\title{
IMPEDANCE OF A LONG SLOT IN A COAXIAL BEAM PIPE*
}

\author{
$\underline{\text { S. De Santis }}^{\#}$, LBNL, Berkeley, CA \\ A. Mostacci, L. Palumbo, Università di Roma "La Sapienza”, Rome, Italy \\ B. Spataro, INFN-LNF, Frascati, Italy
}

\section{Abstract}

We derive an analytical expression for the coupling impedance and loss factor of a long narrow slot in a coaxial beam pipe. The method used differs from the classical Bethe's theory of diffraction since we define differential polarizabilities to take into account the effect of the interference between the fields scattered all along the slot. The expressions obtained are thus valid even for slots longer than the wavelength.

\section{INTRODUCTION}

Different analytical or semianalytical methods can be used to study the effects, on the beam dynamics, of pumping holes and slots coupling the vacuum chamber to an external antichamber.

When the wavelength is much longer than the aperture dimensions, the problem is treated in terms of static polarizabilities and coupling impedance and loss factor can be calculated by different methods $[1,2]$. For longer wavelengths this procedure can no longer be followed and frequency dependant polarizabilities have been introduced in [3].

The method we present here is based on the slot subdivision in infinitesimal slices, as suggested in [4], so that it is still possible to use the modified Bethe's theory of diffraction [5].

\section{GENERAL THEORY}

We consider a long and narrow slot on the inner tube of a coaxial beam pipe (Fig. 1). Subdividing the slot in infinitesimally long elements, which dimensions are much shorter than the wavelength, we can still calculate the equivalent dipole moments for each element according to the modified Bethe's diffraction theory:

$$
\begin{aligned}
d M_{\varphi}(z) & =\left[H_{0 \varphi}(z)-H_{s \varphi}(z)\right] d \alpha_{m} \\
d P_{r}(z) & =\varepsilon\left[E_{0 r}(z)-E_{s r}(z)\right] d \alpha_{e}
\end{aligned}
$$

where $H_{0 \varphi}(z)$ and $E_{0 r}(z)$ are the fields radiated by a point charge $q$, travelling with velocity $c$ along the axis of a perfectly conducting pipe. $H_{s \varphi}(z)$ and $E_{s \varphi}(z)$ are the scattered fields; their amplitude, which is a function of the equivalent dipole moments, can be expressed through the Lorentz reciprocity theorem [2]. The differential

\footnotetext{
*Supported by Department of Energy contract DE-AC03-76SF00098.

\# Email: sdesantis@lbl.gov
}

polarizabilities $d \alpha_{m}$ and $d \alpha_{e}$ are approximated by averaging the static polarizabilities along the slot length $L$ :

$$
d \alpha_{m}=\alpha_{m} / L d z \text { and } d \alpha_{e}=\alpha_{e} / L d z
$$

Limiting our analysis to frequencies below the inner and outer pipes $\mathrm{TE}_{11}$ cutoff, we can rewrite Eqs. (1) as

$$
\begin{aligned}
\frac{d M_{\varphi}}{d z} & =\frac{\alpha_{m}}{L}\left[H_{0 \varphi}-j \frac{\omega \mu h_{0 \varphi}^{2}}{2} \int_{-L / 2}^{L / 2} \frac{d M_{\varphi}}{d \xi} e^{-j k_{0}|z-\xi|} d \xi+\right. \\
& \left.+j \frac{\omega h_{0 \varphi} e_{0 r}}{2} \int_{-L / 2}^{L / 2} \operatorname{sign}(\xi-z) \frac{d P_{r}}{d \xi} e^{-j k_{0}|z-\xi|} d \xi\right] \\
\frac{d P_{r}}{d z} & =\frac{\varepsilon \alpha_{e}}{L}\left[E_{0 r}-j \frac{\omega \mu e_{0 r}^{2}}{2} \int_{-L / 2}^{L / 2} \frac{d P_{r}}{d \xi} e^{-j k_{0}|z-\xi|} d \xi+\right. \\
& \left.+j \frac{\omega \mu h_{0 \varphi} e_{0 r}}{2} \int_{-L / 2}^{L / 2} \operatorname{sign}(\xi-z) \frac{d M_{\varphi}}{d \xi} e^{-j k_{0}|z-\xi|} d \xi\right]
\end{aligned}
$$

where $h_{0 \varphi}$ and $e_{0 r}$ are the TEM modal functions [6] and $k_{0}=2 \pi / \lambda$.
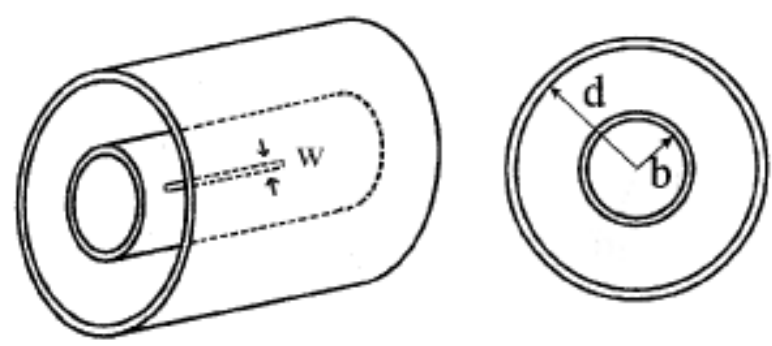

Figure 1: Coaxial beam pipe with slot.

From a physical point of view, Eqs. (3) reveal that the scattered fields depend on the electric and magnetic dipoles all over the aperture, since each infinitesimal slice radiates a forward and a backward wave in the coaxial region [6].

Once Eqs. (3) have been solved, it is straightforward to derive the longitudinal impedance [7]

$$
Z(\omega)=-\frac{1}{q} \int_{-\infty}^{+\infty} E_{z}(r=0) e^{j k_{0} z} d z
$$


Since only the $\mathrm{TM}_{0 \mathrm{~m}}$ modes have a non zero longitudinal electric field along the pipe axis, each element contribution to the impedance is

$$
\frac{d Z}{d z}=j \frac{\omega Z_{0}}{2 \pi q b}\left(\frac{1}{c} \frac{d M_{\varphi}}{d z}+\frac{d P_{r}}{d z}\right) e^{j k_{0} z}
$$

where $Z_{0}$ is the free-space characteristic impedance. Eq. (5) can be regarded as the differential version of the analogous formula derived in [1].

The total impedance is simply obtained integrating Eq. (5) along the slot.

\section{ANALYTICAL EXPRESSIONS FOR IMPEDANCE AND LOSS FACTOR}

To obtain final expressions in an analytical form, we choose to solve the integral equation system in Eqs. (3) using an iterative procedure. It will be shown that it is sufficient to stop at the first order solution.

The zero-th order solution corresponds to the original Bethe's theory [8], appropriately transformed to fit in the integral equations:

$$
\left(\frac{d M_{\varphi}}{d \xi}\right)^{0 t h}=\frac{\alpha_{m}}{L} H_{0 \varphi} \text { and }\left(\frac{d P_{r}}{d \xi}\right)^{0 t h}=\frac{\varepsilon \alpha_{e}}{L} E_{0 r}
$$

Replacing Eqs. (6) in the right hand side of Eqs. (3) we get the first order solution

$$
\begin{aligned}
\left(\frac{d M_{\varphi}}{d z}\right)^{1 s t}= & \left(\frac{d M_{\varphi}}{d z}\right)^{0 t h}+ \\
& -j \frac{\omega}{2} \frac{\alpha_{m}}{L^{2}} \mu H_{0 \varphi}(0) h_{0 \varphi}^{2}\left(\alpha_{m} I_{1}-\alpha_{e} I_{2}\right) \\
\left(\frac{d P_{r}}{d z}\right)^{1 s t}= & \left(\frac{d P_{r}}{d z}\right)^{0 t h}+ \\
& -j \frac{\omega}{2} \frac{\alpha_{e}}{L^{2}} \frac{\mu}{c} H_{0 \varphi}(0) h_{0 \varphi}^{2}\left(\alpha_{e} I_{1}-\alpha_{m} I_{2}\right)
\end{aligned}
$$

where

$$
\begin{gathered}
I_{1}=\int_{-L / 2}^{L / 2} e^{-j k_{0} \xi} e^{-j k_{0}|z-\xi|} d \xi \\
I_{2}=\int_{-L / 2}^{L / 2} \operatorname{sign}(\xi-z) e^{-j k_{0} \xi} e^{-j k_{0}|z-\xi|} d \xi
\end{gathered}
$$

The second order approximation is obtained replacing the expressions found for the differential dipole moments in Eqs. (7) on the right hand side of Eqs. (3). Thus obtaining

$$
\begin{aligned}
& \left(\frac{d M_{\varphi}}{d z}\right)^{2 n d}=\left(\frac{d M_{\varphi}}{d z}\right)^{1 s t}+ \\
& +\frac{\omega^{2}}{4} \mu^{2} \frac{\alpha_{m}}{L^{3}} h_{0 \varphi}^{4} H_{0 \varphi}(0)\left[-\alpha_{m}\left(\alpha_{m} I_{11}-\alpha_{e} I_{12}\right)+\right. \\
& \left.+\alpha_{e}\left(\alpha_{e} I_{21}-\alpha_{m} I_{22}\right)\right] \\
& \left(\frac{d P_{r}}{d z}(z)\right)^{2 n d}=\left(\frac{d P_{r}}{d z}(z)\right)^{1 s t}+ \\
& +\frac{\omega^{2}}{4} \mu^{2} \frac{\alpha_{e}}{c L^{3}} h_{0 \varphi}^{4} H_{0 \varphi}(0)\left[-\alpha_{e}\left(\alpha_{e} I_{11}-\alpha_{m} I_{12}\right)+\right. \\
& \left.+\alpha_{m}\left(\alpha_{m} I_{21}-\alpha_{e} I_{22}\right)\right]
\end{aligned}
$$

The integrals $I_{n m}$ are given by

$$
\begin{gathered}
I_{11}=\iint_{\text {slot }} e^{-j k_{0}|z-\xi|} e^{-j k_{0} \zeta} e^{-j k_{0}|\xi-\zeta|} d \zeta d \xi \\
I_{12}=\iint_{\text {slot }} \operatorname{sign}(\zeta-\xi) e^{-j k_{0}|z-\xi|} e^{-j k_{0} \zeta} e^{-j k_{0}|\xi-\zeta|} d \zeta d \xi \\
I_{21}=\iint_{\text {slot }} \operatorname{sign}(\xi-z) e^{-j k_{0}|z-\xi|} e^{-j k_{0} \zeta} e^{-j k_{0}|\xi-\zeta|} d \zeta d \xi \\
I_{22}=\iint_{\text {slot }} \operatorname{sign}(\xi-z) \operatorname{sign}(\zeta-\xi) \\
\times e^{-j k_{0}|z-\xi|} e^{-j k_{0} \zeta} e^{-j k_{0}|\xi-\zeta|} d \zeta d \xi
\end{gathered}
$$

The complete expression of impedance and loss factor using the second order approximation for the differential dipole moments is quite complex and of no easy readability. From Fig. 2 we can see, though, that the difference from the loss factor for a Gaussian bunch of length $\sigma_{z}$ calculated using the first order approximation is minimal.

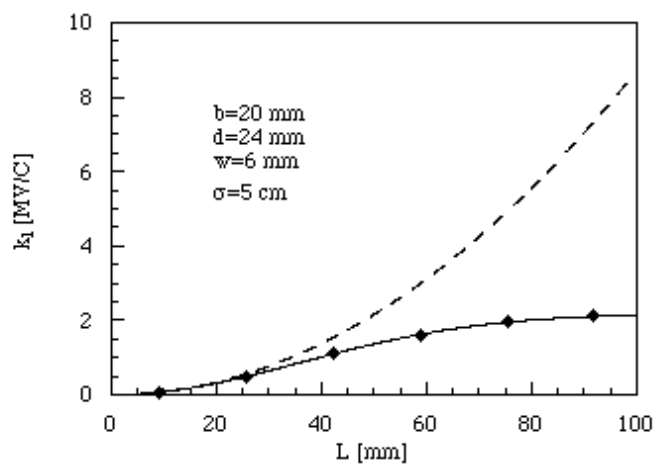

Figure 2: Loss factor vs. length for a rectangular slot. The dashed line is the small aperture approximation [9]; the solid line is the first order solution; the black diamonds are obtained using the second order solution. 
In the following analysis, therefore, we will make use of the following analytical expressions for the longitudinal impedance, obtained using the first order solution of Eqs. (3):

$$
\begin{aligned}
Z_{R E}(\omega)= & \frac{Z_{0} k_{0}^{2}}{32 \pi^{3} b^{4} \ln (d / b)}\left[\left(\alpha_{e}+\alpha_{m}\right)^{2}+\right. \\
& \left.+\left(\alpha_{e}-\alpha_{m}\right)^{2} \frac{1-\cos \left(2 k_{0} L\right)}{2 k_{0}^{2} L^{2}}\right] \\
Z_{I M}(\omega)= & \frac{Z_{0} k_{0}}{4 \pi^{2} b^{2}}\left\{\left(\alpha_{e}+\alpha_{m}\right)+\right. \\
- & \left.\frac{\left(\alpha_{e}-\alpha_{m}\right)^{2}}{8 \pi b^{2} \ln (d / b) L}\left[1-\frac{\sin \left(2 k_{0} L\right)}{2 k_{0} L}\right]\right\}
\end{aligned}
$$

and consequently the loss factor for a Gaussian bunch is

$$
\begin{aligned}
k_{l}\left(\sigma_{z}\right) & =\frac{Z_{0} c \sqrt{\pi}}{128 \pi^{4} b^{4} \ln (d / b) \sigma_{z}} \\
& \times\left\{\frac{\left(\alpha_{e}+\alpha_{m}\right)^{2}}{\sigma_{z}^{2}}+\frac{\left(\alpha_{e}-\alpha_{m}\right)^{2}}{L^{2}}\left[1-e^{-\left(L / \sigma_{z}\right)^{2}}\right]\right\}
\end{aligned}
$$

\section{COMPARISONS WITH NUMERICAL RESULTS}

We have performed simulations with the numerical code MAFIA in the case of both rectangular and rounded end slots of different length and width.

To account for the finite wall thickness $T$ that must be used in the simulations, Eqs. (11) and (12) must be slightly modified as shown in [9]. The electric and magnetic polarizabilities change as well and can be represented as a function of the zero-thickness expressions, using the approximation developed by McDonald [10], as:

$$
\begin{gathered}
\tilde{\alpha}_{e}=C_{E} \alpha_{e} e^{-\pi T \sqrt{1 / L^{2}+1 / w^{2}}} \\
\tilde{\alpha}_{m}=C_{M} \alpha_{m} e^{-\pi T / w}
\end{gathered}
$$

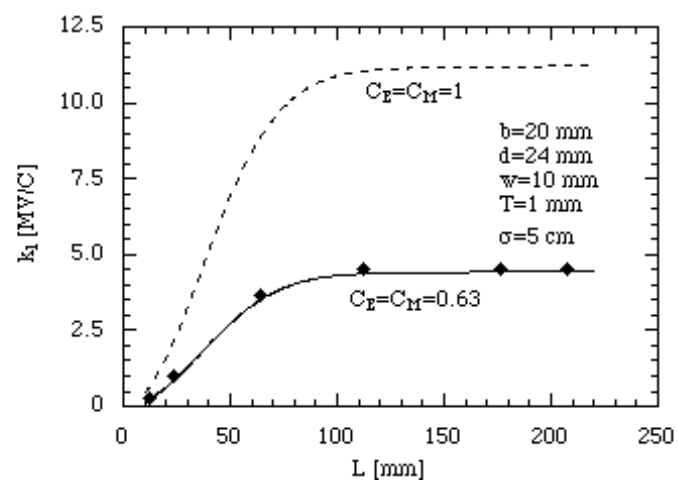

Figure 3: Loss factor for a rectangular slot. The black diamonds are MAFIA points.
Though the $C_{E}$ and $C_{M}$ values are known only for a circular aperture, in our case, a comparison of the analytical (Fig. 3, dashed line) and numerical results suggest the following values: $C_{E}=C_{M}=0.63$.

In order to check this result, the loss factor has been computed numerically for a given slot length and different wall thicknesses (Fig. 4), obtaining $C_{E}=C_{M}=0.62$ as best fit.

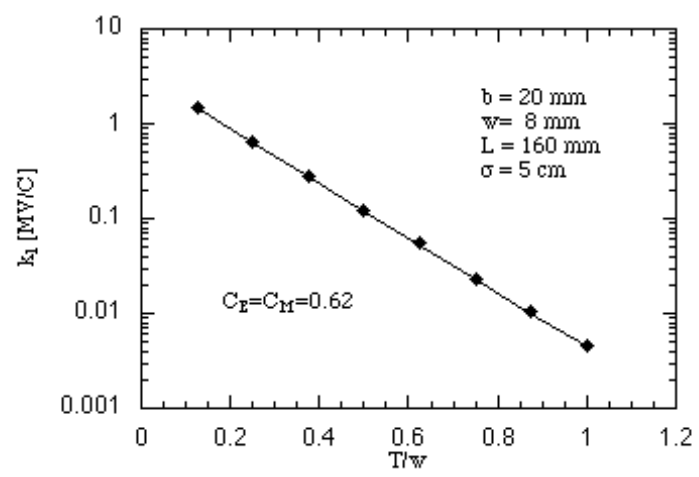

Figure 4: Loss factor for a rectangular slot vs. $T / w$ ratio. The black diamonds are MAFIA points.

\section{CONCLUSIONS}

We have obtained an approximated analytical expression for longitudinal impedance and loss factor of a long narrow slot in a coaxial beam pipe. When the slot is longer than the wavelength, the real part of the impedance shows a typical resonant behaviour related to the slot length. Our results are in good agreement with those obtained in literature with different methods and with MAFIA simulations.

\section{REFERENCES}

[1] S. S. Kurennoy, Part. Accel. 39,1 (1992).

[2] S. De Santis, et al., Phys. Rev. E 54, 800 (1996).

[3] A. Fedotov and R. L. Gluckstern, Phys. Rev. E 54, 1930 (1996).

[4] G. V. Stupakov, Phys. Rev. E 51, 3515(1995).

[5] R. E. Collin, Field Theory of Guided Waves, 2nd ed. (IEEE, New York, 1991).

[6] S. De Santis, et al., Phys. Rev. E 56, 5990 (1997).

[7] L. Palumbo, et al., in CAS Advanced Accelerator School, p. 331 (CERN, Geneva, 1995).

[8] H. A. Bethe, Phys. Rev. 66, 163 (1944).

[9] S. De Santis, et al., Phys. Rev. E 58, 6565 (1998).

[10] N. A. McDonald, IEEE Trans. Microwave Theory Tech. 20, 689 (1972). 